\title{
Didácticas para los procesos de enseñanza-aprendizaje en la práctica formativa en Hemostasia
}

\section{Didactics for the Teaching-Learning Processes in the Training Practice in Hemostasis}

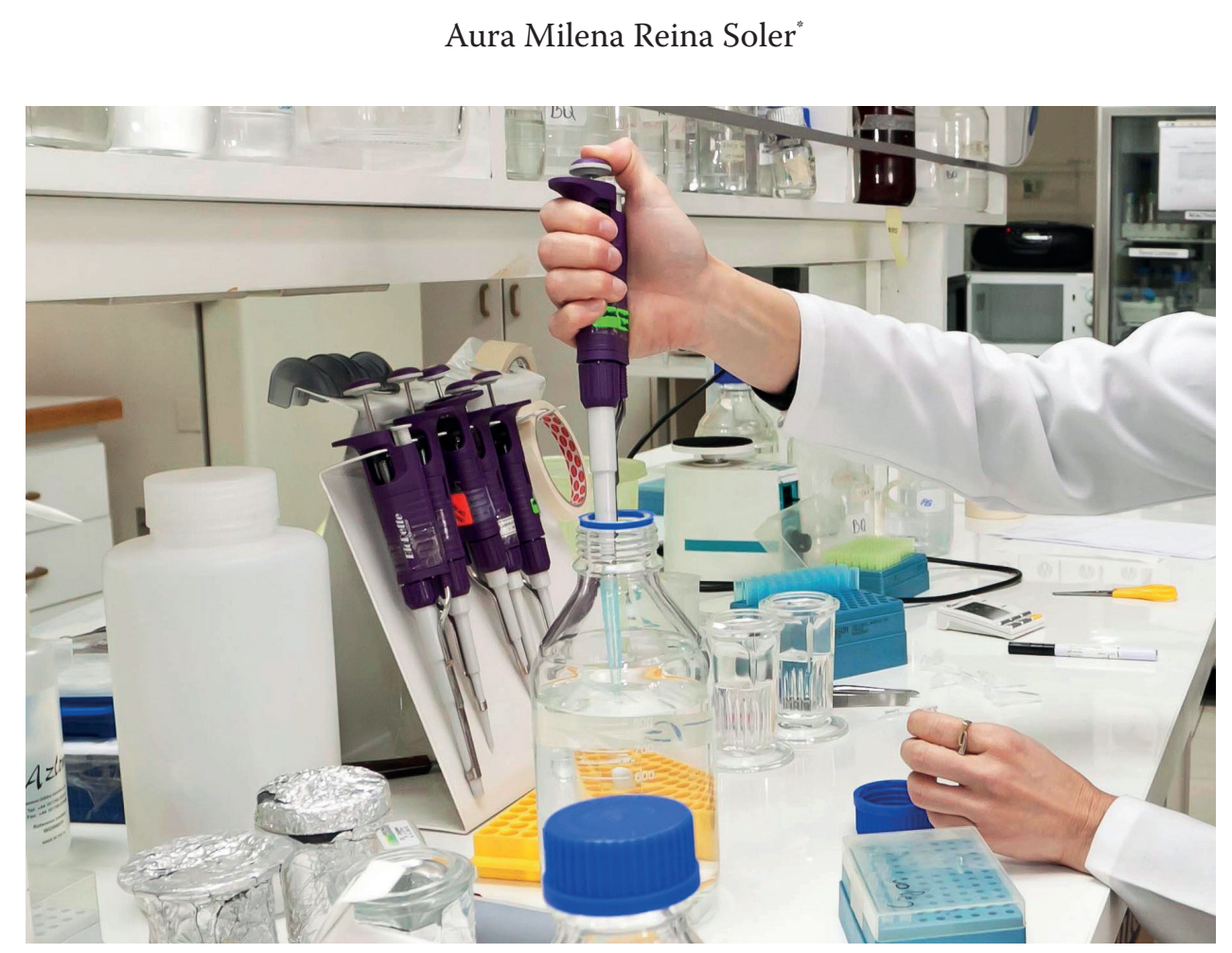

\section{Resumen}

El desarrollo de pruebas diagnósticas para la detección de alteraciones de la hemostasia, su interpretación y correlación requiere del estudio permanente y detallado de los procesos bioquímicos, fisiológicos y patológicos. Los factores que entran a participar en los procesos de enseñanza-aprendizaje de la hemostasia dependen de la complejidad de las temáticas, los preconceptos, las dinámicas aplicadas, la estructuración

Citar este artículo como: Reina Soler, A. M. (2020). Didácticas para los procesos de enseñanza-aprendizaje en la práctica formativa en Hemostasia. Revista Papeles, 12(24), 67-76.

Fecha de recibido: agosto 30 de 2020. Fecha de aceptado: noviembre 10 de 2020.

* Bacterióloga, especialista en hemostasia, especialista en auditoría y gerencia en salud, especialista en docencia universitaria. Docente Universitaria en la Fundación Universitaria de Ciencias de la Salud, Universidad Militar Nueva Granada. Correo electrónico de contacto: mibac_88@hotmail.com 
del plan de trabajo, la metodología y el tiempo requerido para la aprehensión del conocimiento. En este trabajo se aplicó un rango de modalidades educacionales que incluyeron didácticas especiales, actividades de demostración y aprendizaje experimental ligado a la didáctica general. A partir de la literatura revisada y al acercamiento con los residentes, a través del convenio docencia-servicio entre el Laboratorio de Referencia en Hemostasia con la Universidad Militar Nueva Granada y la Fundación Universitaria de Ciencias de la Salud, se consideró establecer cómo las didácticas de enseñanza-aprendizaje de la hemostasia se articulan en la orientación de la praxis para la potenciación de los conocimientos en un área compleja de la medicina.

Se aplicó una encuesta a médicos residentes de hematología y hemato-oncología, rotantes en el período de 2013 a 2016 con el fin de establecer la percepción sobre las didácticas aplicadas en la rotación especializada de hemostasia, las cuales, en un 92\%, demostraron ser adecuadas para la formación de competencias específicas. Es así como se propone la aplicación de didácticas que permitan la articulación de la praxis y la teoría compleja para la potenciación de los conocimientos y el fortalecimiento, construcción y reproducción del aprendizaje.

Palabras clave: medicina, hematología, hematoocología, hemostasia, enseñanzaaprendizaje, didáctica.

\section{Abstract}

The development of diagnostic tests for the detection of alterations of hemostasis, their interpretation and correlation requires the permanent and detailed study of the biochemical, physiological and pathological processes. The factors that enter to participate in the teaching-learning processes of hemostasis depend on the complexity of the topics, the preconceptions, the applied dynamics, the structuring of the work plan, the methodology and the time required for the apprehension of knowledge. In this work, a range of educational modalities was applied a range of educational modalities that included special didactics, demonstration activities and experimental learning linked to general didactics. From the literature reviewed and the approach with the resident physicians through the teaching-service agreement between the Laboratorio de Referencia en Hemostasia with the Universidad Militar Nueva Granada and the Fundación Universitaria de Ciencias de la Salud, it was considered to establish how teaching didactics-learning of hemostasis is articulated in the orientation of praxis for the enhancement of knowledge in a complex area of medicine. A survey of hematology and hematopoietic oncology to resident physicians was carried out in the period from 2013 to 2016 in order to establish the perception about didactics applied in the specialized rotation of hemostasis, which in $92 \%$ were shown to be adequate for the Training of specific skills. Thus the application of didactics that allow the articulation of praxis and complex theory for the empowerment of knowledge and the strengthening, construction and reproduction of learning is proposed.

Key words: medicine, hematology, hematooncology, hemostasis, teaching-learning, didactics. 


\section{Introducción}

La hemostasia se entiende como el proceso fisiológico a través del cual se mantiene la integridad de los vasos sanguíneos y su flujo, en una serie de procesos bioquímicos complejos en los que participan diferentes moléculas con características y funciones esenciales, las fallas de origen congénito o adquirido conducen al deterioro del funcionamiento normal y a alteraciones conocidas dentro de la hematología benigna.

El desarrollo de pruebas diagnósticas para la detección de alteraciones de la hemostasia, su interpretación y correlación, requieren del estudio permanente y detallado de los procesos bioquímicos, fisiológicos y patológicos que permitan realizar diagnósticos acertados, así como el control y seguimiento de los mismos para la toma de decisiones que mejoren la calidad de vida de los pacientes y su seguridad frente a los diferentes procedimientos médicos.

Estas pruebas requieren de centros de referencia especializados, por lo cual el Laboratorio de Referencia en Hemostasia fue creado en el año 1997 con el propósito inicial de contribuir al desarrollo y estandarización de pruebas que contribuyeran al rápido diagnóstico e intervención sobre las patologías hematológicas benignas. Posteriormente, gracias al reconocimiento de la institución, se conviertió en el centro de entrenamiento para especialistas en hematología en el área de estudio.

A partir de la literatura revisada y gracias al acercamiento con los residentes de la Universidad Militar Nueva Granada y la Fundación Universitaria de Ciencias de la Salud, a través de los convenios docencia-servicio con el Centro de Referencia en Hemostasia se observa que en las actividades académicas desarrolladas actualmente, en ejes fundamentalmente clínicos y prácticos, existe una necesidad colectiva sobre la fundamentación teórica en un aprendizaje guiado y la aplicación de didácticas especiales como parte del soporte de formación. Las especializaciones en hematología y hematooncología tienen un componente de hemostasia que requiere de procesos de enseñanza-aprendizaje estructurados en un modelo organizado, consecuente, dinámico y contextualizado que permita, en el tiempo asignado al módulo, la apropiación del conocimiento específico para la complementación de las actividades clínicas. De esta manera, los elementos de enseñanza se deben considerar desde la necesidad de los estudiantes en relación con las metodologías, los recursos, los contenidos, la complejidad y especialidad de las temáticas, las particularidades cognitivas, la intencionalidad social y el saber del docente.

La didáctica reconocida gracias a Juan Amós Comenius (1592-1670) ha sido estudiada por varios autores con aportes desde diferentes perspectivas, con lo cual han establecido sus propias definiciones. En la didáctica magna se reconoce la importancia del aprendizaje a través de la realización de tareas lejos de la ejecución memorística que pretende la reflexión sobre el conocimiento adquirido en un ámbito teórico-práctico. La didáctica puede estructurarse como un conjunto que incluye la enseñanza, la instrucción, la comunicación y la reflexión, es decir, a través de la didáctica se hace saber, según lo detalla Carvajal (2009).

La didáctica comprende los aspectos situacionales educativos, las oportunidades para la transformación del saber, conocidos como trasposición, el proceso de aprendizaje con sus múltiples cambios y los parámetros establecidos entre el docente y el estudiante. Las relaciones establecidas en los procesos de enseñanza-aprendizaje se dan en un contrato didáctico con aspectos positivos relevantes que han sido descritos por Anderson, Bould y Sampson (1996). En general se trata de 
una metodología de enseñanza-aprendizaje "personalizada" que permite dirigir el trabajo independiente o presencial del estudiante promoviendo su autonomía y su capacidad de aprender (Carvajal, 2009, p. 8). En síntesis, la interacción didáctica es un tipo especializado de conversación que implica razonamiento interpersonal entre profesor y alumnos que tiene por finalidad promover aprendizajes (Velasco, 2007). La elección de las estrategias didácticas depende de las habilidades del docente y la disposición para el descubrimiento de las mejores metodologías en la práctica docente y en los diferentes procesos de enseñanza, partiendo del reconocimiento de las habilidades, conocimientos particulares de los estudiantes y los obstáculos ontológicos, didácticos y epistemológicos identificados por Brousseau (1986). Las prácticas didácticas interrelacionan los contenidos de aprendizaje establecidos y los contextualizan en las habilidades, capacidades, destrezas, conocimientos y necesidades de los estudiantes, por lo cual se requiere del compromiso docente y de una planeación acertada. Estas didácticas son exitosas en la medida en que se logre utilizar posteriormente la información con un nivel de reflexión que aporte a la resolución de problemas reales, es decir, cuando logran realizar el proceso de aprendizaje en etapas que les permita comprender, asimilar y aplicar lo aprehendido.

Según Howard, SK., Gaba, DM., Fish, KJ., Yang, G., Sarnquist, FH. Y Weaver, SJ, Dy, SM.,

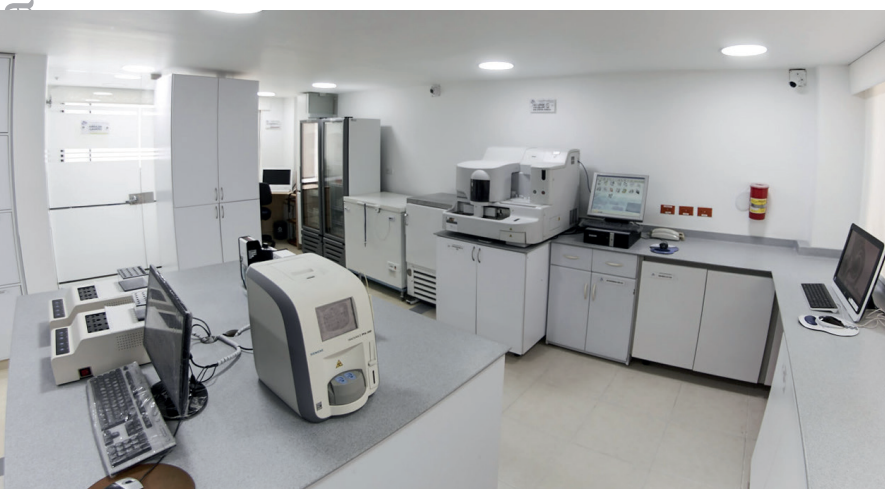

Rosen, MA. en el entrenamiento práctico de las especialidades médicas se usa un rango de modalidades educacionalesque incluyen didácticas formales basadas en lecturas, interacción en grupos y videos; la demostración, como la aplicación de videos especializados y habilidades de trabajo en equipo, y el aprendizaje experimental estudiado en actividades de simulación con tecnologías reales sobre procedimientos estandarizados sin instrucciones previas. La mayor atención sobre la salud del paciente y la regulación de horas laborales para los residentes han hecho de la formación simulada, definida como un ejercicio de aplicación teórico-práctico sobre casos reales ejecutados a través de herramientas tecnológicas, un complemento atractivo a la didáctica tradicional y al aprendizaje clínico (Semler et al. 2015, p. 513). Este estudio realizado en la Universidad de Vanderbilt, Nashville, Tennessee, Estados Unidos, concluye que el aprendizaje basado en la demostración y la simulación muestra mejores resultados que la didáctica tradicional y permite el desarrollo de habilidades para el trabajo en equipo.

De acuerdo con lo anterior, el reconocimiento de la didáctica es un ejercicio de concientización propio de los docentes en la búsqueda del saber que se pretende. En este aspecto Grisales afirma lo siguiente:

La didáctica universitaria también es una interpretación, es decir, una puesta en práctica del saber, tanto disciplinar como didáctico que se comunica en la clase. Razón por la cual, este proceso sistémico debe ser dirigido por docentes con una sólida formación, no sólo en los conocimientos científicos y artísticos propios del saber, sino también en los aspectos correspondientes a su didáctica y al manejo de las diversas variables que caracterizan la enseñanza (Grisales, 2012, p. 216).

La educación didáctica en los programas de salud incluye pasantías, trabajo de campo 
y práctica clínica como mecanismos para la vinculación de la educación y la práctica en procesos reflexivos que conducen a la comprensión de los conceptos y al desarrollo de las competencias necesarias, estos componentes son esenciales incluyendo el laboratorio clínico (Lekkas et al. 2007, Rounce et al. 2007).

En cuanto a didáctica se refiere, es necesario considerar la relevancia de la aplicación de metodologías didácticas en áreas complejas de la medicina. Es así como se propone en este trabajo determinar cuáles son los componentes necesarios para la enseñanza-aprendizaje de la hemostasia con los que se logre potenciar los conocimientos adquiridos hacia la toma de decisiones diagnósticas asertivas en un proceso sistemático y dinámico. Por esta razón, se espera que el uso de didácticas pedagógicas conjuntas minimice el impacto sobre las variables observadas en el proceso de enseñanza-aprendizaje de la hemostasia como en la interacción docente-alumno, en la interrelación de las temáticas, la integralidad de la información, el contexto y la aplicación del saber, los modelos didácticos que sustentan el proceso y la trayectoria formativa del docente. Estas variables comprenden el soporte sobre el cual se fundamenta el ejercicio docente en el Centro de Referencia en Hemostasia, Bogotá (Colombia).

La didáctica es conocida en el arte de la enseñanza desde la Edad Media como un método universal. Según Juan Amós Comenio (1952-1970), probablemente provenga de las necesidades de los estudiantes, quienes perciben la didáctica como un modo práctico de aprendizaje que les facilita el acercamiento al conocimiento de un área especializada. Según Comenio (2007), este método de enseñanza posibilita un tratamiento del conocimiento para su entendimiento, lo que de alguna manera requiere de maestros sabios que sepan compartir y comunicar su sabiduría. Friederich Paulsen (1906) significa el quehacer del docente universitario desde dos frentes, el arte de la ciencia y el arte del saber, en los que se destaca el estímulo sobre los estudiantes para el aprendizaje y la reafirmación del sentido académico.

El método de enseñanza puede interferir en la forma como se adquiere el conocimiento, por lo que según Fernando de los Ríos (1997), la didáctica se divide en dos: general y especial, "la primera trata del plano de la enseñanza y del procedimiento de la misma, [mientras que] la didáctica especial trata de la enseñanza de las especialidades". A mitad del siglo XX, la didáctica estableció un vínculo con la educación superior; así, la didáctica universitaria es considerada como una didáctica especial que se circunscribe a los procesos de enseñanza en el nivel superior de la educación, pero que se sirve -cuando es necesario- de la didáctica general y de las didácticas específicas para comprender su objeto de estudio (Camilioni, 2008).

La teoría y la práctica, por lo tanto, están integradas y los resultados educativos son reconocidos a través de actividades de reflexión intencionales que enlazan los objetivos de aprendizaje con la experiencia del servicio (Bringle y Hatcher, 1999, y Eyler, 2002). Las actividades educativas son necesariamente un complemento que comprende una serie de estrategias para el aprendizaje centrado en la adquisición de conocimientos duraderos y la administración adecuada de los saberes en el ejercicio clínico. Es imperativo desarrollar una estructura que provea el orden necesario en los procesos de enseñanza-aprendizaje para el desarrollo de las capacidades interpretativas y correlativas imprescindibles en la toma de decisiones acertadas. Frente a las alteraciones de la hemostasia se encuentra relevante establecer si la aplicación de didácticas, en principio, es un aspecto relevante en el entendimiento de las temáticas de orden complejo y si con mayor precisión aplica en los ámbitos teóricos o prácticos. 


\section{Metodología}

Para el desarrollo de este trabajo se realizó, inicialmente, una revisión bibliográfica alrededor de referentes contextuales y conceptuales sobre la pregunta planteada con el fin de generar una primera aproximación al campo de estudio; en un segundo momento, siguiendo un enfoque cuantitativo, conocido como aquel que permite la percepción de la realidad de una manera objetiva y descriptiva de hechos sociales y opiniones individuales mediante una significación estadística de la relación causal y su intensidad (López, 2013), se elaboró un instrumento tipo encuesta dirigida a 11 residentes del fellow en hematología de adultos y hematooncología pediátrica, quienes realizaron la práctica formativa en hemostasia en el Centro de Referencia en Hemostasia Bogotá (Colombia) durante el período de 2013 a 2016. La encuesta se aplicó en el primer semestre de 2017 y su propósito era establecer la percepción alrededor de las didácticas de enseñanza aplicadas en el servicio como soporte a las actividades complementarias teóricas y prácticas planteadas en el contenido programático de la rotación: fundamentación teórica especializada, revisión de literatura científica, estudio de caso, correlación clínica e interpretación de resultados, seminario, taller teórico, taller práctico y asistencia a pacientes.

\section{Resultados}

La rotación tiene en promedio 3 residentes por año en períodos de 15 a 90 días según la profundización establecida en el programa de cada facultad. Las preguntas dieron origen a respuestas de tipo cuantitativo en escala de Likert de cinco puntos, donde 1 era poco significativo y 5 muy significativo; las reflexiones fueron resumidas en los aspectos relevantes para el objeto del estudio en los cuales los residentes debían expresar sus preferencias de acuerdo con las didácticas aplicadas en los ámbitos interdisciplinar e interprofesional. Dos preguntas demográficas especificaron el estatus académico y el tiempo de rotación e incluyeron residentes de los niveles I, II y III de subespecialización de dos escuelas de formación diferentes.

Las encuestas fueron analizadas de manera retrospectiva, las cuales mostraron, inicialmente, que en un $92 \%$ se consideraron como actividades que permitían fortalecer los fundamentos teórico-prácticos y afianzar el conocimiento de manera conjunta. La motivación necesaria para el desarrollo de las competencias profesionales fue generada a través de la integración de las didácticas, se observó que las actividades de tipo clínico participativo como el análisis de caso y la asistencia a pacientes obtuvieron el mayor grado de calificación en la escala Likert 5/5. Dentro de las variables de influencia en la aprehensión de los ejes temáticos, el tiempo de rotación mostró que en promedio se requieren de 52 días de rotación para el adecuado desarrollo del plan de trabajo, ya que la experiencia de los docentes fue de $5 / 5$, la didáctica de $5 / 5$ y los recursos de $5 / 5$; los elementos que soportan en mayor grado el aprendizaje de la hemostasia. De otro modo, las herramientas para el aprendizaje consideradas más eficaces son el mapa mental, la interpretación y la correlación clínica, se observa además, que la clase magistral y la inducción teórica son parte del componente de aprendizaje y que en una calificación en escala de $4 / 5$ es necesaria como parte del trabajo guiado. El proceso memorístico no se considera como factor contribuyente al proceso de enseñanza-aprendizaje en este campo, ya que se requiere de la conceptualización a través de la integración coherente en un contexto teórico-práctico. La clase magistral y el seminario como actividades para el 
afianzamiento del conocimiento fueron calificados en escala de 4/5. La revisión de artículo científico, el seminario y el taller teórico en un promedio de 4/5 como didácticas que contribuyen a la motivación muestran una pequeña inferioridad sobre aquellas que incluyen elementos de tipo práctico. El aprendizaje autónomo y la rotación con otros residentes en un mismo ciclo formativo fueron expresados como elementos de menor influencia (4/5) sobre el proceso de enseñanza-aprendizaje, al igual que la inducción teórica y el banco de preguntas como herramientas de aprendizaje.

El uso de herramientas audiovisuales como material didáctico para el desarrollo de las actividades formativas es rescatado como un elemento de aprendizaje y reconocimiento visual que contribuye al entendimiento conceptual especializado con las herramientas de elaboración adecuadas que logren el objetivo para el cual fueron creadas.

Tabla 1.

\section{Percepción de los residentes sobre las didácticas aplicadas para la aprehensión del conocimiento}

Casos clínicos
Considerada por los residentes una de las mejores
formas de aprendizaje, fundamental para la
integración y acercamiento a los casos problé-
micos reales que soportan la toma adecuada de
decisiones a través de la integración multidisci-
plinar del aprendizaje teórico y práctico.

\section{Asistencia a pacientes}

La asistencia a pacientes completa los ejercicios didácticos realizados durante la rotación, permite la integración de todos los elementos y se considera el reflejo o revelación del proceso de enseñanza aprendizaje.

\section{Taller teórico}

Los talleres teóricos han ayudado a la complementacion de los saberes previos; pero además, al reconocimiento de las debilidades específicas que logra el afianzamiento de los conceptos con la retroalimentación oportuna. Es un apoyo a los procesos de correlación en cuanto se genere la integración del conocimiento.

\section{Revisión de artículos}

La actualización de los conceptos específicos basados en la evidencia científica contribuyen a la profundización en áreas particulares del conocimiento, fortalece conocimientos previos o crea nuevas interrelaciones como denomina Ausubel, inclusores obliterativos.

\section{Seminario}

El aprendizaje a través de la aplicación de seminarios se considera un adecuado espacio de profundización en temas de interés particular o de mayor complejidad, sin embargo, se rescta que es una actividad que requiere tiempo para la preparación y es más provechosa cuando se da en un entorno dinámico y participativo.

\section{Taller práctico}

Los talleres prácticos son complementarios y transversales a todas las didácticas, de tal manera que, componen parte fundamental del aprendizaje en hemostasia, en este espacio se permite plasmar el resultado de la estructuración mental dada por otras didácticas con enfoques más teóricos.

\section{Clase magistral}

La percepción frente al ejercicio magistral es positiva en un 81,8\% del total de encuestados. En el plan de trabajo actual, la magistralidad es un espacio de interacción fundamentado en la experiencia del tutor para la resolución de dudas y la ilustración de temas de alta complejidad que son difíciles de estructurar mediante aprendizaje autónomo. Del 81,8\% el 18,17\% está a favor, reconociendo que en algún porcentaje la información no es recordada del todo, o en otros casos, no se logra comunicar la información completa. El 18\% definitivamente no considera el uso de la didáctica magistral ya que prefieren otros modelos de aprendizaje.

Fuente: elaboración propia 


\section{Discusión}

Actualmente, siete tipos de didáctica hacen parte de la enseñanza en el Centro de Referencia en Hemostasia, los resultados obtenidos son positivos y responden a didácticas que construyen y potencian los conocimientos, pero es importante reflexionar sobre la aplicación de un modelo de integración didáctica, o por el contrario, en la individualización de las mismas, teniendo en cuenta el eje temático, su complejidad y las necesidades de conceptualización particulares. Los procesos de memorización considerados por los residentes como no significativos para los procesos de aprendizaje en este estudio generan un punto de quiebre en cuanto a la concepción de los orígenes teóricos y la evolución de los mismos; por lo tanto, los conceptos básicos de la hemostasia se retan al desarrollo sobre los

\section{Los elementos teóricos, aunque} son parte fundamental en el curso de los procesos educativos, resultan ser menos atractivos e influyentes en la enseñanza-aprendizaje de la hemostasia, probablemente debido

a la intensidad y complejidad de las temáticas que requieren estructuras dinámicas para una mejor comprensión. contextos que fortalezcan el reconocimiento de ambos extremos en el ámbito clínico. Los elementos teóricos, aunque son parte fundamental en el curso de los procesos educativos, resultan ser menos atractivos e influyentes en la enseñanza-aprendizaje de la hemostasia, probablemente debido a la intensidad y complejidad de las temáticas que requieren estructuras dinámicas para una mejor comprensión. Ahora bien, es preciso reconocer si se requieren de otras didácticas o mayores estructuraciones que permitan modular de igual manera la aprehensión del conocimiento en diferentes niveles de profesionalización y en diferentes grados de preconcepción.

Las didácticas, de acuerdo con lo referido por los encuestados, se pueden subdividir según las pretensiones del programa, los residentes $y$ las competencias que se requieren fortalecer, según los objetivos planteados al inicio de cada ciclo de rotación, lo cual crea nuevos espacios de estudio y seguimiento. Se espera concretar si las experiencias didácticas reflejan el verdadero sentido de la construcción del conocimiento en un trabajo continuo en el cual se integran diferentes tipos de aprendizaje: cooperativo, experiencial, interdisciplinario, cognitivo, basado en equipos y por cumplimiento de objetivos, entre otros, o son parte de un todo en un lugar con mayor o menor grado de importancia, teniendo claro que la integración de las didácticas produce una mayor capacidad de interrelación y evita los procesos monótonos que dilatan el aprendizaje y afectan la ecología del aula.

\section{Conclusiones}

Los procesos educativos convergen actualmente en la progresión de los procesos de enseñanza-aprendizaje a través de la utilización de diferentes herramientas que desarrollan procesos cognitivos, cognoscitivos y metacognitivos; cada uno de estos factores se enlaza en una estructura sólida que permite la formación de saberes específicos; pero además, superiores e integrales con una intencionalidad social. La importancia de la aplicación de didácticas de enseñanza especiales en un área subespecializada 
influirá de manera positiva en la construcción, integración y reproducción del conocimiento, con lo cual se demostraría en la práctica los avances en las impresiones diagnósticas acertadas y oportunas que se traducen en la implementación adecuada de tratamientos y en la costo-efectividad del sistema de salud.

La oportunidad para la construcción de un saber específico está relacionada, en primer lugar, con la intencionalidad del estudiante, con lo cual la labor docente deberá enfocarse en el tipo de aprendizaje, las didácticas necesarias, los recursos para la enseñanza y el contexto sobre el cual se debe vincular a los estudiantes para su profesionalización.

Este trabajo, finalmente, invita a profundizar sobre cómo los métodos de enseñanza y el dominio sobre una especialidad médica bajo un sólido estado del arte nos lleva a una apropiación mayor del conocimiento y su transformación gracias a la aplicación de didácticas a los ámbitos teóricos y prácticos. De acuerdo con Tomaschewsky (1996):

considerar otros elementos como los fines y objetivos de la enseñanza, las leyes y principios del proceso, los contenidos y la planeación en actividades articuladas pueden mejorar y facilitar las experiencias académicas de los especialistas en un ámbito complejo y muy limitado.

La experiencia del docente, basada en los aspectos laborales-profesionales y aquellos adquiridos a través de la actualización continua para el dominio de un saber, argumenta los procesos de enseñanza-aprendizaje asociados al cumplimiento de los objetivos y alineados al currículo, los métodos, las didácticas y los procedimientos; sin embargo, la autonomía del docente constituye un componente principal en el desarrollo de las habilidades y las competencias en una disciplina en la que se deben generar las estrategias de comunicación con los educadores y los educandos para la socialización de las experiencias, las competencias actuales y el alcance en niveles de educación especializada como actividades que conjuntamente imparten el estímulo necesario sobre las acciones educativas hacia el mejoramiento continuo del aprendizaje. Basados en este estudio se puede afirmar que la didáctica constituye parte de una estrategia global para el desarrollo adecuado de una educación especializada, pero también es parte de un proceso motivacional que fortalece el aprendizaje, su construcción y reproducción en áreas específicas del conocimiento, la aplicación de la didáctica en salud cualifica y orienta la praxis del ejercicio docente.

\section{Referencias}

Anderson, G., Boud. D., Sampson, J. 1996. Qualities of Learning contracts. Higher education for capability.

Bringle, R., Hatcher, J. 1999. Reflection in Service-Learning: Making of Experience. Educational Horizons, 179-185.

Brousseau, G. 1983. Los obstáculos epistemológicos y los problemas en matemáticas. Revista de educación matemática, 4(2), 164-198.

Carvajal, M. 2009. La didáctica. Fundación Academia de Dibujo Profesional.
Camilloni, A. 2008. Didáctica general y didácticas específicas. El saber didáctico. Buenos Aires: Paidós, 23-39.

Comenio, J. A. 1971. Didáctica Magna $2^{\circ}$ ed. Madrid: Reus S.A.

Comenio, J. A. 2000. Didáctica Magna $11^{\circ}$ ed. México: Porrúa.

Comenio, J. A. 2007. Didáctica Magna $17^{\circ}$ ed. México: Editorial Porrúa.

De los Ríos, F. 1997. Obras completas III: escritos breves. Barcelona: Anthropos. 
Eyler, J. 2002. Reflection: Linking Service and Learning-Linking Students and Communities.

Social Issues, 58(3), 517-534. doi: 10.1111/ 1540-4560.00274.

Grisales-Franco, L.M. 2012. Aproximación histórica al concepto de didáctica universitaria. Educ, 15(2), 203-218.

Howard, SK., Gaba, DM., Fish, KJ., Yang, G., Sarnquist, FH. Anesthesia crisis resource management training: teaching anesthesiologists to handle critical incidents. Aviat Space Environ Med, 1992(63), 763-770.
Lekkas, P., Larsen, T., Kumaar, S., Grimmer, K., Nyland, L., Chipcase, L., Finch, J. (2007), "No model of clinical education for physiotherapy students is superior to another: a systematic review". Aust. Journal of Physiotherapy, 53(1), 19-28.

López, L. 2013. Enfoque cuantitativo de la investigación. Ministerio de Educación, Universidad pedagógica experimental Libertador. Venezuela-El tigre.

Paulsen, F., Thilly, F., \& Elwang, W. 1906. The german universities and university study. New York: Bibliolife Network. 